\title{
Dynamical landscape in DODAB membrane system: MD simulation \& neutron scattering studies
}

\author{
H. Srinivasan, V.K. Sharma, S.Mitra, V. Garcia Sakai \\ and R. Mukhopadhyay
}

\section{Published version information}

Citation: H Srinivasan et al. "Dynamical landscape in DODAB membrane system: MD simulation \& neutron scattering studies." Physica B, vol. 562 (2019): 55-58.

DOI: $10.1016 /$ i.physb.2018.12.013

(C)2019. This manuscript version is made available under the CC-BY-NC-ND 4.0 Licence.

This version is made available in accordance with publisher policies. Please cite only the published version using the reference above. This is the citation assigned by the publisher at the time of issuing the AAM. Please check the publisher's website for any updates. 


\title{
Dynamical landscape in DODAB membrane system: MD Simulation \& Neutron Scattering Studies
}

\author{
H. Srinivasan ${ }^{1}$, V.K. Sharma ${ }^{1}$, S.Mitra ${ }^{1,2^{*}}$, V. Garcia Sakai ${ }^{3}$ and R.Mukhopadhyay ${ }^{1,2}$ \\ ${ }^{1}$ Solid State Physics Division, Bhabha Atomic Research Centre, Trombay, Mumbai - 400085 , \\ India \\ ${ }^{2}$ Homi Bhabha National Institute, Anushaktinagar, Mumbai 400094, India \\ ${ }^{3}$ ISIS Pulsed Neutron and Muon Facility, Science and Technology Facilities Council, Rutherford \\ Appleton Laboratory, Didcot OX11 0QX, U.K. \\ *smitra@barc.gov.in
}

\begin{abstract}
The nature of diffusion of lipids in a bilayer membrane plays a key role in various biological processes like cell signaling, cellular transport etc. Dioctadecyldimethylammonium bromide (DODAB) is a synthetic lipid that self-assembles to form bilayer. Here we describe the dynamical landscape of the DODAB bilayer in fluid phase using molecular dynamics (MD) simulation and quasielastic neutron scattering (QENS) techniques. MSD of lateral motion of the lipids calculated from MD simulations show subdiffusive behaviour. A model free approach is employed to describe the dynamics of lipids in the bilayer where the relaxation rates are distributed over a wide range. A stretched exponential relaxation function used to describe the incoherent intermediate scattering function of the lipids obtained from MD simulation and QENS experiments. The distribution of relaxation rates covers over three decades. The results of the MD simulation and QENS experiments agree well.
\end{abstract}

\section{Introduction}

Dioctadecyldimethylammonium bromide (DODAB) is a cationic lipid [1] that self assembles into various structures like vesicles, bilayers etc. when dissolved in an aqueous solvent. The selfassembled structures of DODAB are found to show rich phase behaviour with four different polymorphic phases [2-5]. In the increasing order of alkyl chain ordering, the four polymorphic phases are given - coagel, subgel, gel and fluid. At low concentrations ( $<0.5 \mathrm{wt} \%)$ subgel phase is formed at room temperature, which upon heating transforms into gel phase and further heating causes significant increase in alkyl chain ordering transforming into the fluid phase. At higher concentrations (> $6 \mathrm{wt} \%$ ), at room temperature the system is found to be in the coagel phase and by heating directly transforms into the fluid phase. However, while cooling, fluid phase transforms into an intermediate gel phase before transforming into the coagel phase. Fourier 
Transform Infrared Spectroscopy (FTIR) [2, 3] on DODAB dispersions suggest that the coagel and subgel phases have highest degree of alkyl chain ordering, while the fluid phase is highly disordered due to significant number of gauche defects. Further, in the fluid phase, the broadening of modes corresponding to $\mathrm{CH}_{2}$ stretching indicates that there is an increase in chain mobility. The lamellar spacing in different phases have been measured using small angle $\mathrm{X}$-ray scattering [2, 3] and shown to be the lowest in coagel and highest in fluid suggesting a relationship between local alkyl chain ordering and lamellar packing of bilayers.

Quasielastic neutron scattering (QENS) has been employed to study DODAB bilayer in coagel, gel and fluid phases [6] with an aim to probe the dynamical transitions and diffusion mechanisms. It has been found that lateral motion of lipids is frozen in the coagel phase, while it's activated in the gel and fluid phases [6]. QENS spectra in the coagel phase are well described considering only the localised motion of the alkyl chains. But in the fluid and gel phases along with the motion of alkyl chains, the lateral motion of lipids is also present and is found to be explained in the framework of Fickian diffusion. However, it has been observed that lateral motion of the lipids in various bilayer systems, exhibit subdiffusive nature [7-9]. Therefore, to gain molecular insight on the mechanism of lateral diffusion of lipids in the DODAB bilayer, we carry out molecular dynamics (MD) simulation of the DODAB bilayer in aqueous media. QENS and MD simulation probe the dynamics of the molecules in the almost the same spatial and temporal regimes and provide complementary details of the system. In this work we attempt to describe the dynamical landscape of the DODAB lipid motion without assuming a particular model for the diffusion mechanism of the DODAB lipids.

\section{Materials and methods:}

DODAB $\left(\mathrm{C}_{18} \mathrm{H}_{37}\right)_{2} \mathrm{~N}\left(\mathrm{CH}_{3}\right)_{2} \mathrm{Br}$ powder (purity > 98\%) and $\mathrm{D}_{2} \mathrm{O}$ (99.9\% atom D purity) were obtained from Tokyo Chemical Industries Co. LTD. and Aldrich respectively. We have used the same neutron scattering data as reported in ref. [10]. Neutron scattering experiments on DODAB dispersion and $\mathrm{D}_{2} \mathrm{O}$ were carried out at $345 \mathrm{~K}$ using IRIS spectrometer at the ISIS pulsed Neutron and Muon source at the Rutherford Appleton Laboratory, UK. IRIS spectrometer was used with PG (002) analyser in the offset mode providing a resolution of $\sim 17 \mu \mathrm{eV}$ with energy transfer range from -0.3 to $1.0 \mathrm{meV}$ [11]. In order to minimise the scattering contribution of the solvent, $\mathrm{D}_{2} \mathrm{O}$ was used in preparing the DODAB dispersion. $70 \mathrm{mM}$ DODAB dispersion was prepared by magnetically stirring the mixture DODAB and $\mathrm{D}_{2} \mathrm{O}$ at a temperature of $\sim 340 \mathrm{~K}$ until a clear solution was formed. Separate QENS experiments were also carried out on $\mathrm{D}_{2} \mathrm{O}$ to estimate the solvent contribution using the same sample cell. The QENS spectra of the DODAB bilayer was obtained by subtracting the solvent $\left(\mathrm{D}_{2} \mathrm{O}\right)$ contribution [12],

$$
S_{\text {bilayer }}(Q, E)=S_{\text {solution }}(Q, E)-\varphi S_{D_{2} O}(Q, E)
$$


where $\varphi$ is the volume fraction of solvent in the solution.

Trajectories obtained from MD simulation performed earlier [10] are used for this analysis as well. Initially, two monolayers of DODAB molecule were constructed with 64 monomers in each monolayer. Subsequently, 3600 solvent water (TIP3P) molecules were added in a box above and below the bilayer. Simulation was carried out within a cubic box of length of 64 A. Interaction parameters were obtained from CHARMM force field [13], but the bonds associated with hydrogen atoms in DODAB were kept fixed. Particle-Mesh Ewald summation technique was used for long-range interactions with a real space cut-off of $10 \AA$. MD simulations were carried out at $350 \mathrm{~K}$, with a pressure of $1 \mathrm{~atm}$. The system was initially equilibrated for 15 ns and followed by a 4 ns production run. NPT ensemble was used with Langevin barostat and thermostat. The trajectories were recorded at an interval of 4 ps. DL_Poly_4 [14], was used to carry out the MD simulations. To obtain the short time trajectories, a separate run of 20 ps was also carried out and trajectories were recorded with an interval of $0.02 \mathrm{ps}$.

\section{Results and discussion:}

The MD simulation of DODAB bilayer in aqueous medium at $350 \mathrm{~K}$ resulted in the fluid phase of the bilayer system. The system equilibration was effectively concluded after the area per lipid (APL) of the bilayer converged to a value of $\sim 64 \AA^{2}$. The fluid phase of the lipid bilayer was marked by significant number of gauche defects in the alkyl chain. Fig. 1 shows the snapshot of the fluid phase of DODAB lipid bilayer; two lipid molecules are also highlighted to indicate the disorder in the alkyl chain. We investigated the lateral motion of lipids in the bilayer using the results of MD simulation and found it to be subdiffusive in nature. A model free approach is being considered to analyse the QENS spectra of the DODAB bilayer and compared with results of MD simulation.

\subsection{MD simulation - subdiffusion of lipids}

In order to investigate the lateral motion of the lipids in the bilayer plane, we calculate the mean squared displacement (MSD) of the lipid centre of mass (COM) from MD simulation trajectories. The lateral component of the MSD is calculated using the $(x, y)$ coordinates of the lipid COM. Fig. 2(a) shows the MSD of lateral motion, it is found that the lateral MSD is proportional to $t^{2}$ in the ballistic regime that continues upto $\sim 0.1 \mathrm{ps}$. This is followed by a subdiffusive regime, where it scales as $t^{\alpha}$; the subdiffusive exponent, $\alpha$, can be explicitly calculated using the equation,

$$
\left\langle\delta r_{\text {lat }}^{2}(t)\right\rangle=A t^{\alpha} \quad \Rightarrow \quad \alpha(t)=\frac{d\left(\ln \delta r_{\text {lat }}^{2}(t)\right)}{d(\ln t)}
$$


The variation of the subdiffusive exponent, $\alpha$, with time, clearly showed that the motion of lipids is not Fickian in nature but follow a subdiffusive law with an exponent of $\sim 0.62$ [10]. The variation of the subdiffusive exponent with respect to time is shown in Fig. 2(b). The subdiffusive motion and subdiffusive exponent, $\alpha(t)$, has been associated to the crowding of the lipid molecules in the bilayer $[8,9,10]$. The crowding of lipids in the bilayer is known to lead to some memory effects giving rise to a non-Markovian diffusion process. This has been characterised in detail considering an asymptotically power law memory function in the generalised Langevin equation for velocity autocorrelation function [10].

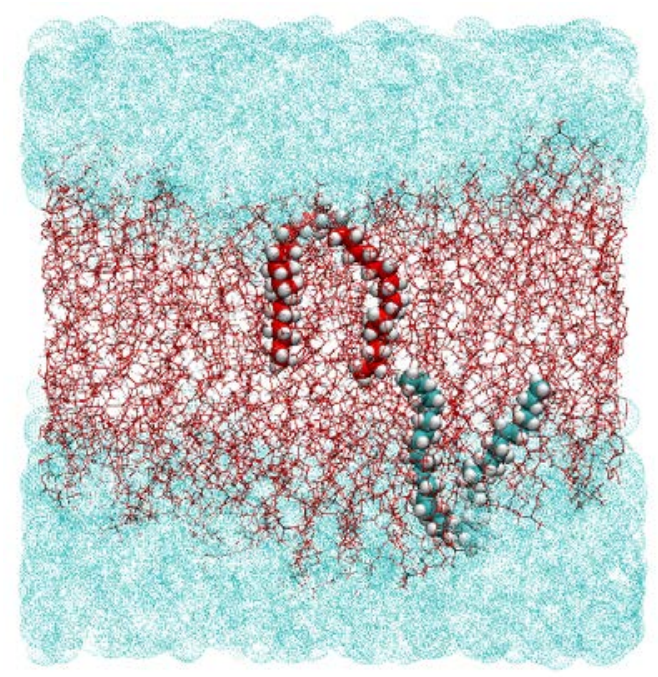

Figure 1 Snapshot of the DODAB lipid bilayer system in the fluid phase (350 K).

\subsection{QENS experiments - Hydrogen mobility}

In neutron scattering experiments, the double differential cross-section gives the probability that a neutron is scattered in a solid angle element $(d \Omega)$ with an energy exchange between $E$ and $E+d E$, given by [15]

$$
\frac{d^{2} \sigma}{d \Omega d E}=\frac{k_{i}}{k_{f}}\left[\sigma_{c o h} S_{c o h}(\boldsymbol{Q}, \omega)+\sigma_{i n c} S_{i n c}(\boldsymbol{Q}, \omega)\right]
$$

where, $k_{i}, k_{f}$ are the momenta of neutron before and after scattering respectively, $\sigma_{c o h}, \sigma_{\text {inc }}$ are the coherent and incoherent scattering cross-sections, and $S_{\text {coh }}, S_{\text {inc }}$ are the coherent and incoherent scattering laws for the system. The scattering laws are given as functions of $\boldsymbol{Q}$ and $\omega$ that are the momentum and energy transfer respectively in the neutron scattering process. Hydrogen has a large $\sigma_{\text {inc }}$ in comparison to the coherent or incoherent scattering cross-sections of other atoms. Therefore, the neutron scattering spectra from a hydrogenous material is dominated by 
incoherent contribution from hydrogen atoms. The incoherent scattering law is the Fouriertransform of the self-part of van-Hove correlation function $G_{s}(\boldsymbol{r}, t)$. Intermediate incoherent scattering function (IISF), $I_{\text {inc }}(Q, t)$, can be related to the incoherent part of neutron spectra by,

$$
I_{i n c}(Q, t)=\int_{-\infty}^{\infty} S_{i n c}(Q, \omega) e^{i \omega t} d \omega
$$

where, $Q=k_{f}-k_{i}$ and $\hbar \omega=E_{f}-E_{i}$ are the momentum and energy transfer of the neutron respectively. IISF can be calculated from MD simulations using the trajectories of all hydrogen atoms in the system, thereby providing a bridge between QENS experiments and MD simulation. Since, hydrogen atoms have an overwhelming contribution to the incoherent spectra, we have calculated the $I_{\text {inc }}(Q, t)$ considering only the hydrogen atoms in the system. The calculation of $I_{\text {inc }}(Q, t)$ from simulation trajectories is carried out using time-origin average,

$$
I_{\text {inc }}(Q, t)=\frac{1}{N} \sum_{j=1}^{N} \overline{\left\langle e^{-i Q \cdot r_{j}\left(t_{0}\right)} e^{i Q \cdot r_{j}\left(t+t_{0}\right)}\right\rangle}
$$

where, $\boldsymbol{r}_{\boldsymbol{i}}(t)$ is the position of the $i^{\text {th }}$ hydrogen atom, $t_{0}$ is an arbitrary time-origin and $\mathrm{N}$ is the total number of hydrogen atoms. While the brackets denote average over time-origins, the bar denotes average over all $Q$-orientations to resemble powder-averaging.

In light of the subdiffusive behaviour of lipid COM as observed in MD simulation and discussed in the last section, we can expect a similar nature of diffusion in hydrogen atoms. Nevertheless, unlike the lateral motion of lipid COM the motion of hydrogen atoms is a superposition of various degrees of freedom inclusive of the internal motion of the alkyl chain and lateral motion of the lipids. Therefore, to begin with, we compute the MSD of all hydrogen atoms from MD simulation, $\left\langle\delta r_{H}^{2}(t)\right\rangle$, and observe that it is subdiffusive in nature,

$$
\left\langle\delta r_{H}^{2}(t)\right\rangle=B t^{\beta} \quad \Rightarrow \quad \frac{d\left(\ln \delta r_{H}^{2}(t)\right)}{d(\ln t)}=\beta
$$



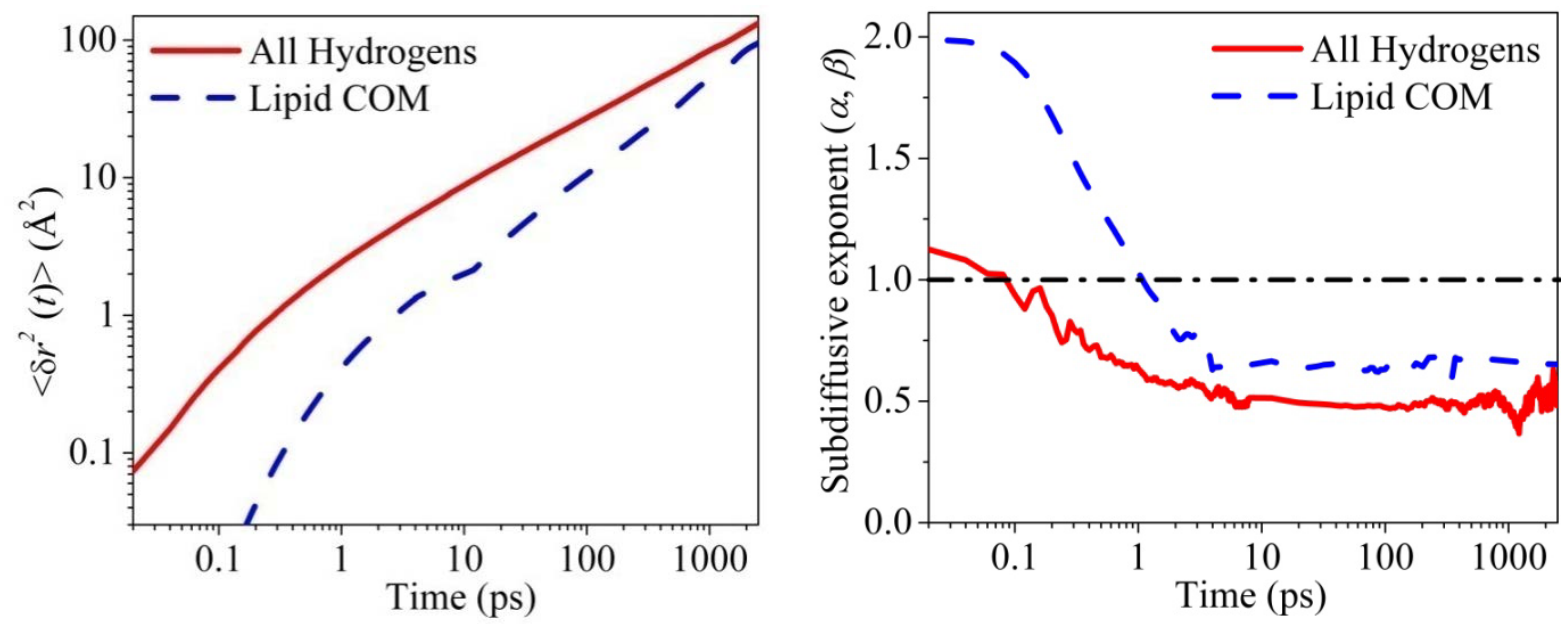

Figure 2 (a) Variation of MSD of lipid centre of mass for all hydrogen atoms in the DODAB lipid bilayer. (b) The corresponding subdiffusive exponents, $\alpha(t)$ and $\beta(\mathrm{t})$, calculated from equations (2) and (6) for lipid COM and all hydrogen atoms respectively.

From Fig. 2(b), it is clear that the motion of hydrogen atoms show a subdiffusive behaviour with $\beta \sim 0.5$ for $t>5$ ps. In the region $\mathrm{t}>5 \mathrm{ps}$, fitting $\left\langle\delta r_{H}^{2}(t)\right\rangle$ with $B t^{\beta}$, gives $\beta \sim 0.5$ and $B \sim 2.4$ $\AA^{2} / \mathrm{ps}^{\beta}$.

As noted earlier, since the motion of hydrogen atoms is a combination of various degrees of freedom, Gaussian approximation cannot be used to relate MSD and IISF of hydrogen atoms. Therefore, we consider a model-independent approach where the timescale of relaxation motion of hydrogen atoms are widely distributed. A stretched exponential [16] of the following form is used to analyse the IISF obtained from QENS spectra (eq. 4) and MD simulation (eq. 5),

$$
I_{H}(Q, t)=\exp \left[-\left(t / \tau^{*}\right)^{\chi}\right]
$$

where $\tau^{*}$ and $\chi$ are the fitting parameters. Fig. 3 (a) shows the fits based on eq. 7 to IISF (for $\mathrm{t}>$ 5 ps) obtained from QENS spectra (eq. 4) and MD simulation (eq. 5) indicating that stretched exponential describes the experimental and simulation IISF reasonably well. The stretching parameter, $\chi$, from the fit is found to be $\sim 0.5$. Fig. 4(a) shows the obtained values of $\chi$ at two different Q-values from fits on QENS spectra and MD simulation results. The stretched 

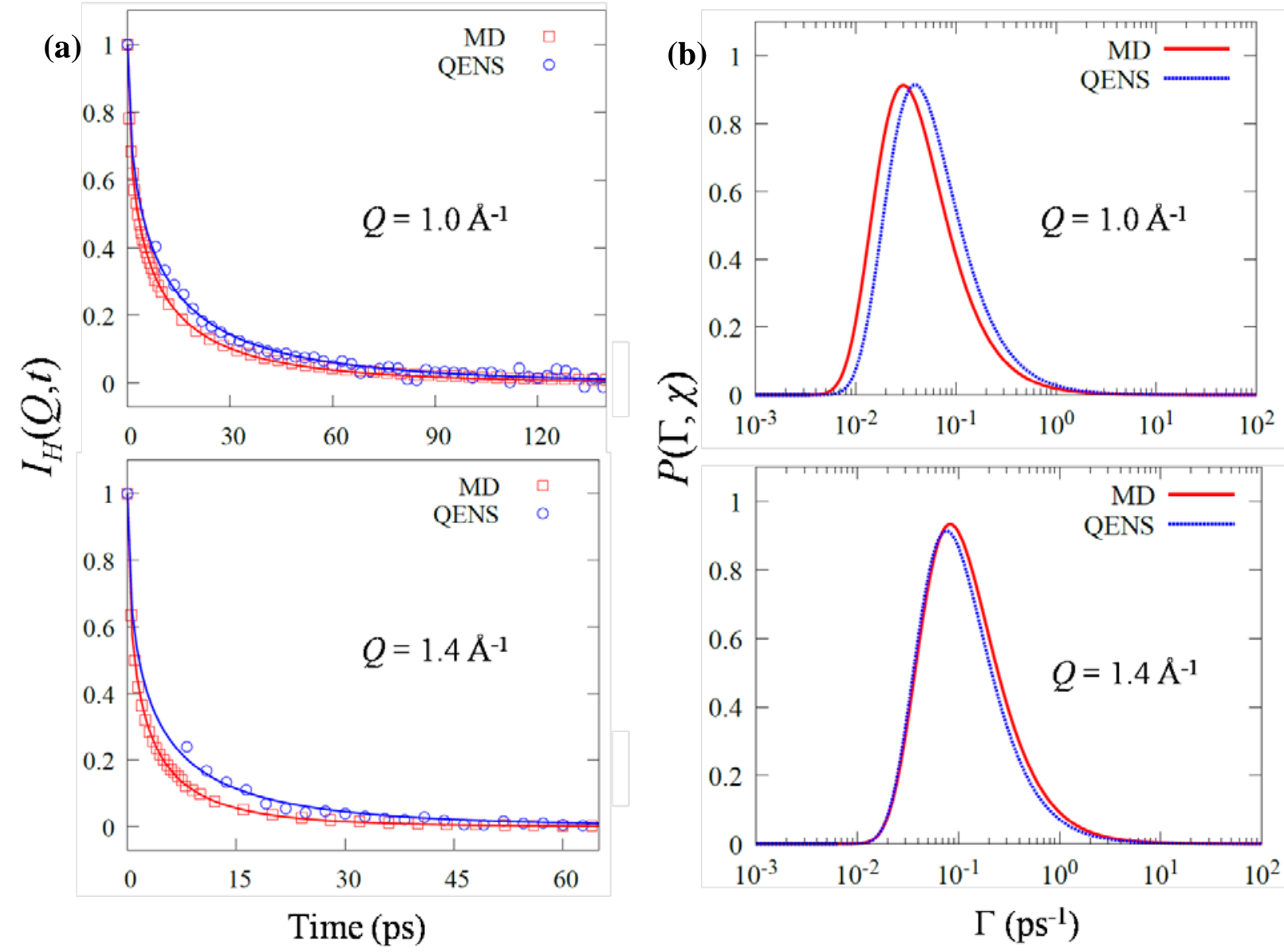

Figure 3 (a) IISF of the DODAB bilayer calculated from QENS spectra and MD simulation shown along with their fits based on eq. (7). (b) The distribution of relaxation rates calculated from eq. (9) using the fit parameters obtained from the fit using eq. (7).

exponential function can be expressed as a continuous sum of exponential relaxation processes distributed over a wide range of timescales [16],

$$
I_{H}(Q, t)=e^{-\left(t / \tau^{*}\right)^{\chi}}=e^{-\left(\lambda^{*}\right)^{\chi}}=\frac{1}{\lambda *} \int_{0}^{\infty} P(\lambda, \chi) e^{-\lambda t} d \lambda
$$

Here, $\lambda^{*}$ is the inverse of relaxation time $\tau^{*}$ and $P(\lambda, \chi)$ is the distribution of rates of relaxation $(\lambda$ $=1 / \tau)$ in the system. For a given $\chi(0<\chi \leq 1)$, the probability distribution, $P(\lambda, \chi)$ is normalized over the relaxation rates, $\lambda$. For $\chi=1$, the distribution corresponds to Dirac-delta function, giving rise to single exponential relaxation with the relaxation rate, $\lambda=\lambda^{*}$. The deviation of the exponent $\chi$ from 1 indicates the existence of a distribution of relaxation rates in the system. The distribution function can be numerically calculated using an infinite series summation given by $[16,17]$, 


$$
P(\lambda, \chi)=\frac{1}{\pi} \sum_{n=0}^{\infty} \frac{(-1)^{n+1} \Gamma(n \chi+1)}{n ! \lambda^{n \chi+1}} \sin (n \pi \chi)
$$

where, $\Gamma(x)$ is the gamma function. The distribution of rates of relaxation calculated from the fit parameters of eq. (8) is shown in Fig. 3 (b) for two typical $Q$-values. Closed form expressions for rational, $\chi=n / m$, can be obtained in terms of generalized hypergeometric functions [16]. The series approximation for the distribution (eq. (9)) is known to be quickly convergent, however poses serious problems in the calculation for values of $\lambda<\lambda^{*}$, where the series takes on extremely large values due to error in the limited precision [17]. This was overcome by using the 'Decimal' package in python that allows us to define real numbers of arbitrary precision. In this calculation a precision of 60 digits and upto 400 terms in the series expansion were considered to get the distribution function over the complete range. The lipids in the bilayer can undergo various kinds of motion including lateral diffusion of lipid along the bilayer, localized diffusion of the segments in the alkyl chain etc. It is clearly observed that the relaxation rates are distributed over three decades indicating that it might arise from different degrees of freedom of the lipids. An excellent agreement between the distribution calculated from QENS spectra and MD simulation is also observed. However, an average timescale for the $I_{H}(Q, t)$ based on the model used in eq. (8), the average timescale is given by [16],

$$
\langle\tau\rangle=\frac{\tau^{*}}{\chi} \Gamma\left(\frac{1}{\chi}\right)
$$
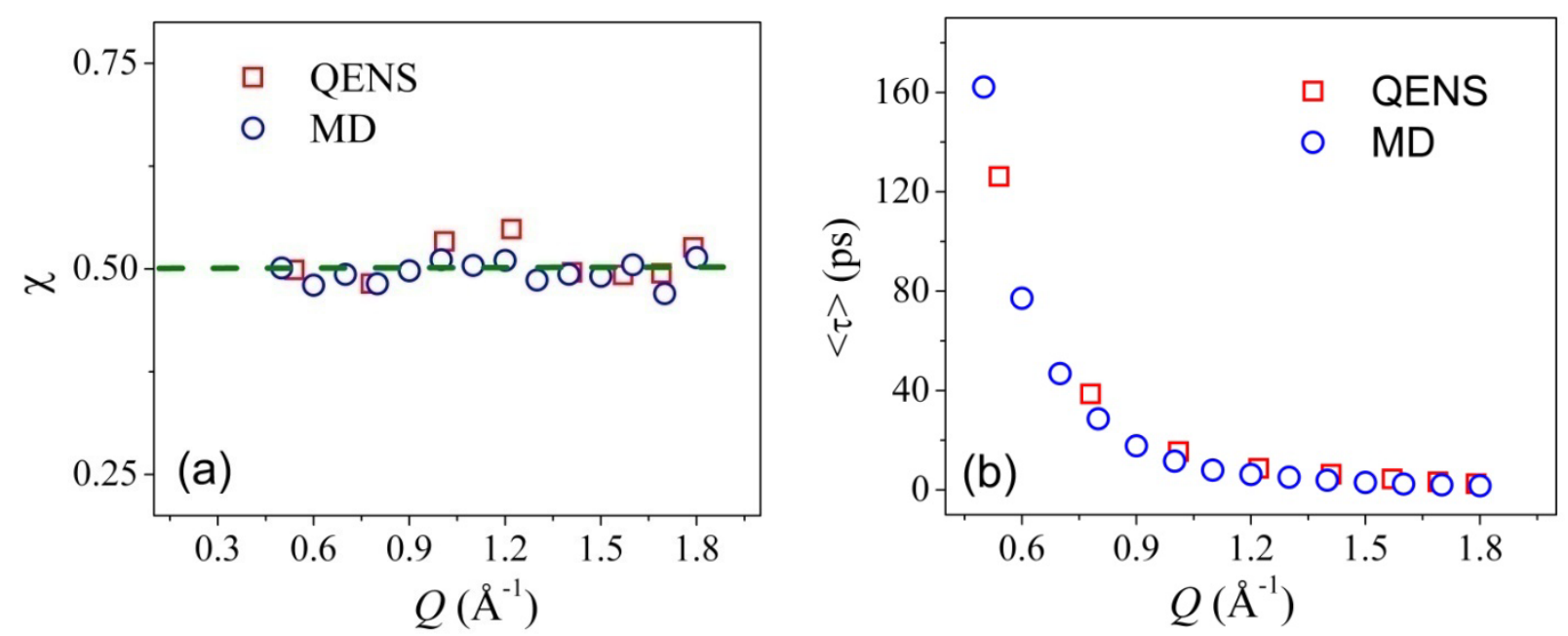

Figure 4 (a) Stretching exponent $\chi$ obtained from fitting IISF of QENS experiment and MD simulation with eq. (7). (b) The corresponding average timescale $<\tau>$ calculated using eq. (10) based on the fits from eq. (7). 
Variation of $\langle\tau\rangle$ is shown in Fig. 4 (b) for both the experimental QENS data and MD simulation results. It is found that the match between the experiment and simulation is very good over the observed $Q$ range.

\section{Conclusion:}

The study of dynamics of DODAB bilayers in the fluid phase using MD simulation reveals that the lateral diffusion of the centre of mass of lipids is subdiffusive in nature. QENS experiments were carried out in order to describe the dynamical landscape of lipids in the bilayer. The MD simulation trajectories showed that the mean squared displacement (MSD) of all hydrogen atoms in the bilayer is also subdiffusive in nature. In order to describe the QENS spectra that arises from the contribution of all the degrees of freedom of lipid dynamics, intermediate incoherent scattering function (IISF) is described considering a wide distribution of timescales. In this model free approach, a stretched exponential function is found to describe the IISF of both QENS experiments and MD simulation very well. The distribution of relaxation rates is observed to span over three decades. This might indicate that the distribution of relaxation rates include contribution from different kinds of motion like lateral motion of lipids and internal motion of alkyl chains. The agreement between results of MD simulation and QENS experiments are quite satisfactory. Therefore, this model free approach for describing the dynamics of DODAB lipids without assuming any particular ansatz works quite satisfactorily for QENS and MD simulation data simultaneously. 


\section{References:}

[1] T. Kunitake, Y. Okahata Journal of the American Chemical Society, 99 (1977) 3860-3861

[2] F.-G. Wu, Z.-W. Yu, G. Ji Langmuir, 27 (2011) 2349-2356

[3] F.-G. Wu, N.-N. Wang, Z.-W. Yu, Langmuir, 25 (2009) 13394-13401

[4] E. Feitosa, P.C.A. Barreleiro, G. Olofsson ,Chemistry and Physics of Lipids, 105 (2000) 201-213

[5] E. Feitosa, R.D. Adati, P. Hansson, M. Malmsten, PLOS ONE, 7 (2012) e44702

[6] P.S. Dubey, H. Srinivasan, V.K. Sharma, S. Mitra, V.G. Sakai, R. Mukhopadhyay, Scientific Reports, 8 (2018) 1862

[7] E. Flenner, J. Das, M.C. Rheinstädter, I. Kosztin, Physical Review E, 79 (2009) 011907

[8] J.-H. Jeon, H.M.-S. Monne, M. Javanainen, R. Metzler, Physical Review Letters, 109 (2012) 188103

[9] R. Metzler, J.H. Jeon, A.G. Cherstvy, Biochimica et Biophysica Acta (BBA) Biomembranes, 1858 (2016) 2451-2467

[10] H. Srinivasan, V.K. Sharma, S. Mitra, R. Mukhopadhyay, The Journal of Physical Chemistry C, 122 (2018) 20419-20430

[11] C.J. Carlile, M.A. Adams, Physica B: Condensed Matter, 182 (1992) 431-440

[12] V.K. Sharma, E. Mamontov, D.B. Anunciado, H. O'Neill, V.S. Urban, Soft Matter, 11 (2015) 6755-6767

[13] R.B. Best, X. Zhu, J. Shim, P.E.M. Lopes, J. Mittal, M. Feig, A.D. MacKerell, Journal of Chemical Theory and Computation, 8 (2012) 3257-3273

[14] W. Smith, C.W. Yong, P.M. Rodger, Molecular Simulation, 28 (2002) 385-471

[15] M. Bée, Quasielastic neutron scattering : principles and applications in solid state chemistry, biology and materials science, A. Hilger, Bristol; Philadelphia, 1988.

[16] D.C. Johnston, Physical Review B, 74 (2006) 184430

[17] C.P. Lindsey, G.D. Patterson, The Journal of Chemical Physics, 73 (1980) 3348-3357 